Advances in Radio Science (2003) 1: 63-66

(C) Copernicus $\mathrm{GmbH} 2003$

\title{
On the matrices of capacitance and inductance coefficients for lossless homogeneous multiconductor transmission lines
}

\author{
A. Reibiger \\ Technische Universität Dresden, Fakultät Elektrotechnik, Professur für Theoretische Elektrotechnik, Mommsenstr. 13, 01062 \\ Dresden, Germany
}

\begin{abstract}
Using results from the theory of planar fields and complex functions it is shown that for lossless homogeneous multiconductor transmission lines the computation of the matrices of capacitance and inductance coefficients can be reduced to the solution of some special Dirichlet boundary value problems. Additionally, a function theoretic proof is given for the relationship $\mathbf{L C}=\epsilon \mu \mathbf{E}$ between these matrices.
\end{abstract}

\section{Introduction}

Besides of rare exceptions the standard textbook representations of transmission line equations for two-conductor transmission lines start with a finite LC-ladder network as a model for a lossless transmission line. The system of transmission line equations is then developed by means of some questionable limit processes.

Let $\Lambda$ denote a multiconductor transmission line consisting of $n+1$ perfect conductors embedded in a perfectly insulating, homogeneous dielectric with permittivity $\epsilon$ and permeability $\mu$. The propagation of TEM waves on $\Lambda$ can be described in terms of voltages and currents by means of the transmission line equations $\frac{\partial \mathbf{u}}{\partial z}=-\mathbf{L} \frac{\partial \mathbf{i}}{\partial t}, \frac{\partial \mathbf{i}}{\partial z}=-\mathbf{C} \frac{\partial \mathbf{u}}{\partial t}$ where $\mathbf{L}$ and $\mathbf{C}$ are the $n \times n$ matrices of inductance and capacitance coefficients, resp. Under the assumption that the matrices $\mathbf{L}$ and $\mathbf{C}$ exist it is possible to derive the transmission line equations from the system of Maxwell's equations. For the special case of two-conductor transmission lines, i.e. for the case $n=1$, such a proof was known to Heaviside (1971) at least in 1883. But it may be that this fact had been known to him even already since 1876 (Heaviside, 1970). As a corollary of such a proof it follows that these matrices obey the relationship $\mathbf{L C}=\epsilon \mu \mathbf{E}$, a fact seemingly first observed by Wagner (1914). Since the columns of the matrix $\mathbf{C}$ can be computed by solving $n$ special Dirichlet boundary value problems this

Correspondence to: A. Reibiger

(reibiger@iee.et.tu-dresden.de) formula is very useful. It allows the computation of $\mathbf{L}$ by means of $\mathbf{L}=\epsilon \mu \mathbf{C}^{-1}$.

The weak point in the just mentioned argumentation is the assumption that the matrix $\mathbf{L}$ of the inductance coefficients exists. Elementary approaches for the computation of $\mathbf{L}$, e.g. application of Biot-Savart's law etc., do not work because (i) the surface current density distribution is unknown and (ii) it is impossible to guarantee that the magnetic field does not penetrate the conductors although they are assumed to be perfectly conducting. But more sophisticated methods are also difficult to apply.

By means of the theory of planar fields and complex function theory (Henrici, 1974; Lawrentjew and Schabat, 1967) we present a direct proof of the identity $\mathbf{L C}=\epsilon \mu \mathbf{E}$. Starting with the above mentioned solutions of $n$ special Dirichlet boundary value problems we construct first of all $n$ complex basis potentials for the set of all transversal electrostatic fields on $\Lambda$. Since the cross section of $\Lambda$ is a multiply connected domain it is necessary for this construction to introduce $n$ suitable branch cuts. The complex potential for any transversal electrostatic field on $\Lambda$ can then be represented as a linear combination of these complex basis potentials. In the next step we show that a simple multiplication of these complex basis potentials with the complex constant $-j$ delivers the complex basis potentials for transversal stationary magnetic fields on $\Lambda$ such that the complex potential of any transversal stationary magnetic field on $\Lambda$ can be represented as a linear combination of the complex basis potentials obtained in this way. Complex contour integrals enclosing the cross sections of the conductors of $\Lambda$ deliver then the elements of the matrices $\mathbf{C}$ and $\boldsymbol{\Gamma}:=\mathbf{L}^{-1}$. A simple coefficient comparision delivers the identity in question. The $n$ branch cuts necessary for a unique definition of the complex potentials are essentially used for the evaluation of the complex contour integrals. Last but not least, a classical result of potential theory for two-dimensional domains guarantees the existence of all our complex basis potentials if the conductor boundaries are sufficiently smooth. 


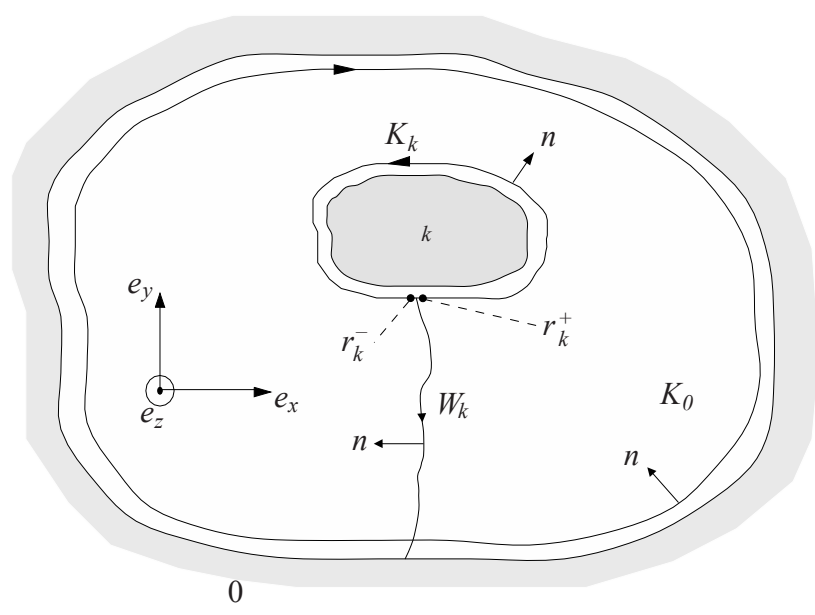

Fig. 1. Graphical representation of the cross section of $\Lambda$.

\section{Matrices of capacitance and inductance coefficients for lossless homogeneous multiconductor transmis- sion lines}

We consider a multiconductor transmission line $\Lambda$ with the following properties:

$\Lambda$ consists of $n$ perfectly conducting cylindric inner conductors with constant cross sections which are parallel to each other and of infinite length. These conductors are enclosed by an perfectly conducting cylindric outer conductor which is also of infinite length.

We assume that the electromagnetic fields do not penetrate these conductors. Therefore, it is only necessary to admit surface current densities and surface charge densities, denoted in the following by $\mathcal{S}$ and $\sigma$, resp. As it is standard we denote the electric field strength by $\mathcal{E}$, the displacement density by $\mathcal{D}$, the magnetic field strength by $\mathcal{H}$, and the magnetic induction by $\mathcal{B}$.

The space between the conductors is filled with a perfect insulating dielectric with permittivity $\epsilon$ and permeability $\mu$.

The conductors of $\Lambda$ are denoted by the natural numbers $0,1, \ldots, n$. The boundary surface between the conductors and the insulator is denoted by $A$, and $v:=1 / \sqrt{\epsilon \mu}$ denotes the light velocity in the insulator material.

Figure 1 shows a cross section of $\Lambda$. For simplicity we have shown in Fig. 1 only the outer conductor, denoted by 0 , and that inner conductor which is denoted by the number $k$. In figure 1 there are also shown the integration paths $K_{0}, K_{k}$, and $W_{k}$ and their normal vectors. For $k=1, \ldots, n$ the path $W_{k}$ starts at conductor $k$ and ends at conductor 0 . The paths $W_{k}(k=1, \ldots, n)$ are pairwise disjoint and and each path $W_{k}$ does not intersect or touch one of the conductors denoted by $l \in\{1, \ldots, n\} \backslash\{k\}$. Subsequently, these paths and normal vectors are used for the definition of voltages and currents. As reference directions for the currents in the conductors $k=1, \ldots, n$ we use the positive orientation of the $z$-axis. Because of the right-hand rule convention included implicitly in Ampère's integral law we have to use the oppositely oriented paths $\left(-W_{k}\right)$ for the computation of the magnetic fluxes per unit length trough the $W_{k}(k=1, \ldots, n)$ by means of the function theoretic methods mentioned in the introduction.

With respect to the coordinate system sketched in Fig. 1 by means of the basic vectors $e_{x}, e_{y}, e_{z}$ we introduce the projection $\Xi$ defined by the assignment $(x, y, z) \mapsto(x, y)$ and the embedding $\Theta$ defined by $(x, y) \mapsto(x, y, 0)$.

For simplicity of representation we assume additionally that the surfaces of the conductors are sufficiently smooth and that all functions considered in the following are at least two times continously differentiable.

Next, we turn towards the determination of the matrices of capacitance and inductance coefficients. These matrices are characteristics of the electrostatic and the stationary magnetic fields on $\Lambda$, resp.

An ordered triple $(\mathcal{E}, \mathcal{D}, \sigma)$ is an electrostatic field on $\Lambda$ if it is a solution of $\operatorname{rot} \mathcal{E}=0, \operatorname{div} \mathcal{D}=0, \mathcal{D}=\epsilon \mathcal{E}, \sigma=n \cdot \mathcal{D} \mid A$, and $n \times \mathcal{E} \mid A=0$.

An ordered triple $(\mathcal{H}, \mathcal{B}, \mathcal{S})$ is an stationary magnetic field on $\Lambda$ if it is a solution of $\operatorname{rot} \mathcal{H}=0, \operatorname{div} \mathcal{B}=0, \mathcal{B}=\mu \mathcal{H}$, $\mathcal{S}=n \times \mathcal{H} \mid A$, and $n \cdot \mathcal{B} \mid A=0$

For the computation of the matrices of capacitance and inductance coefficients we are only interested in $z$-independent transversal solutions of these equations since we need these matrices for a description of TEM waves. Therefore we always assume that the conditions $\mathcal{E}=\left(E_{x}, E_{y}, 0\right)$, $\mathcal{D}=\left(D_{x}, D_{y}, 0\right), \mathcal{H}=\left(H_{x}, H_{y}, 0\right), \quad \mathcal{B}=\left(B_{x}, B_{y}, 0\right)$, and $\mathcal{S}=\left(0,0, S_{z}\right)$ are fulfilled. Thus, nontransversal electrostatic and stationary magnetic fields on $\Lambda$ as discussed in Nähring (2002) are excluded.

By means of $U_{k}:=\int_{W_{k}} \mathcal{E} \cdot \mathrm{d} r \quad(k=1, \ldots, n)$ we assign to each transversal electrostatic field on $\Lambda$ the associated voltage matrix $\mathbf{U}:={ }^{\mathrm{t}}\left(U_{1}, \ldots, U_{n}\right)$. And by means of $I_{k}:=\int_{K_{k}} \mathcal{S} \cdot e_{z} \mathrm{~d} s=\int_{K_{k}}(n \times \mathcal{H}) \cdot e_{z} \mathrm{~d} s=-\int_{K_{k}} \mathcal{H} \cdot\left(n \times e_{z}\right) \mathrm{d} s$ $=\int_{K_{k}} \mathcal{H} \cdot\left(e_{z} \times n\right) \mathrm{d} s$, i.e. $I_{k}:=\int_{K_{k}} \mathcal{H} \cdot \mathrm{d} r \quad(k=1, \ldots, n)$, we assign to each transversal stationary magnetic field on $\Lambda$ the associated current matrix $\mathbf{I}:={ }^{\mathrm{t}}\left(I_{1}, \ldots, I_{n}\right)$.

Now we are able to set up and to prove the following Theorem There are symmetric, positive definite $n \times n$ matrices $\mathbf{C}, \boldsymbol{\Gamma}$ and $\mathbf{L}$ with

$\mathbf{C}=\epsilon \mu \boldsymbol{\Gamma}, \mathbf{L}=\boldsymbol{\Gamma}^{-1}$

such that for each transversal static electric field $(\mathcal{E}, \mathcal{D}, \sigma)$ on $\Lambda$ and its associated voltage matrix $\mathbf{U}$, and for each transversal stationary magnetic field $(\mathcal{H}, \mathcal{B}, \mathcal{S})$ on $\Lambda$ and its associated current matrix $\mathbf{I}$ the conditions

$\oint_{K_{k}} \mathcal{D} \cdot n \mathrm{~d} s=\mathbf{C}_{\mathbf{k} \bullet} \mathbf{U}$

and $\oint_{-W_{k}} \mathcal{B} \cdot n \mathrm{~d} s=\mathbf{L}_{k \bullet \mathbf{I}}$

are fulfilled for each $k=1, \ldots, n$ where $\mathbf{C}_{k}$ and $\mathbf{L}_{k}$ denotes the $k$-th row of the matrices $\mathbf{C}$ and $\mathbf{L}$, resp.

Proof: In the following $G$ denotes the complete image of the space between the conductors of $\Lambda$ under the projection $\Xi$. 
Let $(\mathcal{E}, \mathcal{D}, \sigma)$ be a transversal electrostatic field on $\Lambda$. Because the conductor surfaces are always equipotential surfaces of the $\mathcal{E}$-field the line integrals $\oint_{K_{k}} \mathcal{E} \cdot t \mathrm{~d} s$ are vanishing for all $k=0,1, \ldots, n$. Therefore the potential function of $\Xi \circ \mathcal{E} \circ \Theta$ is globally defined on $G$ and the computation of the corresponding electrostatic field can be reduced to that one of a Dirichlet boundary value problem.

In a first step we consider on $G$ the $n$ special Dirichlet boundary value problems $\Delta_{2} \varphi_{l}=0, \quad \varphi_{l}\left|K_{0}=0, \quad \varphi_{l}\right| K_{l}$ $=\delta_{k l} \quad(k, l=1, \ldots, n)$ where $\Delta_{2}$ denotes the differential operator defined for each $C^{2}$-function $\varphi$ by means of $\Delta_{2} \varphi:=$ $\frac{\partial^{2} \varphi}{\partial x^{2}}+\frac{\partial^{2} \varphi}{\partial y^{2}}$ and $\delta_{k l}$ denotes the so called Kronecker symbol defined for all $k, l=1, \ldots, n$ by $\delta_{k k}:=1$ and $\delta_{k l}:=0(k \neq l)$.

The boundary conditions determining the functions $\varphi_{l}$ $(l=1, \ldots, n)$ imply that the $\varphi_{l}$ are a system of linearly independent functions. Obviously, their linear combination $\varphi=\sum_{l} \varphi_{l} U_{l}$ delivers for each family of voltages $U_{l}$ $(l=1, \ldots, n)$ a solution of the boundary value problem given by $\Delta_{2} \varphi=0, \varphi\left|K_{0}=0, \varphi\right| K_{l}=U_{l} \quad(l=1, \ldots, n)$.

From standard uniqueness arguments it follows then that the $\varphi_{l}(l=1, \ldots, n)$ are not only linearly independent but that they are a basis for the set of the solutions of all these boundary value problems, too.

By means of suitable branch cuts, as such one can be used the curves $W_{l}(l=1, \ldots, n)$, we associate to each function $\varphi_{l}$ a conjugate function $\psi_{l}$. The functions $\psi_{l}$ are then defined on a simply connected subdomain of $G$ which we denote by $G^{*}$.

An ordered triple $(\mathcal{E}, \mathcal{D}, \sigma)$ is a transversal electrostatic field on $\Lambda$ if and only if there exists a family $\left(U_{l}\right)_{l=1, \ldots, n}$ such that the associated planar field $\Xi \circ \mathcal{E} \circ \Theta$ can be represented in either of the two forms $\Xi \circ \mathcal{E} \circ \Theta(r)=-\operatorname{grad}_{2} \varphi(r)$ $(r \in G), \quad \Xi \circ \mathcal{E} \circ \Theta(r)=-\operatorname{rot}_{1} \psi(r) \quad\left(r \in G^{*}\right)$ where $\varphi$ and $\psi$ are the real and imaginary part of a complex potential $\varphi \mid G^{*}+\mathrm{j} \psi$ defined by $\varphi:=\sum_{l} \varphi_{l} U_{l}, \psi:=\sum_{l} \psi_{l} U_{l}$ and the nonstandard differential operators $\operatorname{grad}_{2}$ and $\operatorname{rot}_{1}$ are defined for each scalar $C^{1}$-field $\varphi$ by means of $\operatorname{grad}_{2} \varphi:=\left(\frac{\partial \varphi}{\partial x}, \frac{\partial \varphi}{\partial y}\right)$ and $\operatorname{rot}_{1} \varphi:=\left(\frac{\partial \varphi}{\partial y},-\frac{\partial \varphi}{\partial x}\right)$, respectively.

Let $(\mathcal{H}, \mathcal{B}, \mathcal{S})$ be a transversal stationary magnetic field on $\Lambda$. Because there do not exist magnetic monopols the line integrals $\oint_{K_{k}} \mathcal{B} \cdot n \mathrm{~d} s$ are vanishing for all $k=0,1, \ldots, n$. Therefore the stream function of $\Xi \circ \mathcal{B} \circ \Theta$ is globally defined on $G$. Together with the fact that for $l=1, \ldots, n$ each equipotential line of $-\operatorname{grad}_{2} \varphi_{l}$ is a field line of $-\operatorname{rot}_{1} \varphi_{l}$, and vice versa, this observation motivates for a complex potential $\vartheta+\mathrm{j} \chi \mid G^{*}$ of $\Xi \circ \mathcal{B} \circ \Theta$ the ansatz $\vartheta:=+\sum_{l} \psi_{l} \Phi_{l}$, $\chi:=-\sum_{l} \varphi_{l} \Phi_{l}$ where $\left(\Phi_{l}\right)_{l=1, \ldots, n}$ is a family of flux values per unit length. And indeed, an ordered triple $(\mathcal{H}, \mathcal{B}, \mathcal{S})$ is a transversal stationary magnetic field if and only if there exists a family $\left(\Phi_{l}\right)_{l=1, \ldots, n}$ such that the associated planar field $\Xi \circ \mathcal{B} \circ \Theta$ can be represented by means of the just defined complex potential in either of the two forms $\Xi \circ \mathcal{B} \circ \Theta(r)=$ $-\operatorname{grad}_{2} \vartheta(r)\left(r \in G^{*}\right), \Xi \circ \mathcal{B} \circ \Theta(r)=-\operatorname{rot}_{1} \chi(r)(r \in G)$. Together with the limit values $\Psi_{k l}^{+}:=\psi_{l}\left(r_{k}^{+}\right), \quad \Psi_{k l}^{-}:=$ $\psi_{l}\left(r_{k}^{-}\right)$of the functions $\psi_{l}(l=1, \ldots, n)$ on the left and right edges of the corresponding branch cuts (cf. fig. 1) it follows for the charge $Q_{k}$ per unit length on conductor $k$ and for the current $I_{k}$ in this conductor the relationship

$$
\begin{aligned}
Q_{k} & =\oint_{K_{k}} \mathcal{D} \cdot n \mathrm{~d} s=\sum_{l} \epsilon\left(\Psi_{k l}^{+}-\Psi_{k l}^{-}\right) U_{l}, \\
I_{k} & =\oint_{K_{k}} \mathcal{H} \cdot t \mathrm{~d} s=\sum_{l} \mu^{-1}\left(\Psi_{k l}^{+}-\Psi_{k l}^{-}\right) \Phi_{l},
\end{aligned}
$$

respectively. And with $C_{k l}:=\epsilon\left(\Psi_{k l}^{+}-\Psi_{k l}^{-}\right), \quad \Gamma_{k l}:=$ $\mu^{-1}\left(\Psi_{k l}^{+}-\Psi_{k l}^{-}\right), \quad \mathbf{C}:=\quad\left(C_{k l}\right)_{k, l=1, \ldots, n}, \quad \boldsymbol{\Gamma}:=$ $\left(\Gamma_{k l}\right)_{k, l=1, \ldots, n}$ there results $C_{k l}=\epsilon \mu \Gamma_{k l} \quad(k, l=1, \ldots, n)$, i.e.

$\mathbf{C}=\epsilon \mu \Gamma=\epsilon \mu \mathbf{L}^{-1}$,

$\mathbf{L C}=\epsilon \mu \mathbf{E}$.

What are to be proved.

To understand the physical meaning of the parameters $\Phi_{k l}$ and $\Gamma_{k l}$ let us consider for $k \in\{1, \ldots, n\}$ a family $\left(\Phi_{l}\right)_{l=1, \ldots, n}$ defined by $\Phi_{k} \neq 0$ and $\Phi_{l}=0(l \in\{1, \ldots, n\} \backslash\{k\})$. If we denote the start point of $W_{k}$ by $r_{k}^{\mathrm{s}}$ and the end point of $W_{k}$ by $r_{k}^{\mathrm{e}}$ then

$$
\begin{aligned}
\int_{-W_{k}} \mathcal{B} \cdot n \mathrm{~d} s & =-\int_{-W_{k}} \operatorname{rot}_{1} \chi \cdot n \mathrm{~d} s \\
& =\int_{W_{k}} \operatorname{rot}_{1} \chi \cdot n \mathrm{~d} s \\
& =\chi\left(r_{k}^{\mathrm{e}}\right)-\chi\left(r_{k}^{\mathrm{s}}\right) \\
& =\left(\varphi_{k}\left(r_{k}^{\mathrm{s}}\right)-\varphi_{k}\left(r_{k}^{\mathrm{e}}\right)\right) \Phi_{k} \\
& =\varphi_{k}\left(r_{k}^{\mathrm{s}}\right) \Phi_{k} \\
& =\Phi_{k} .
\end{aligned}
$$

Thus $\Phi_{k}$ is equal to the flux per unit length through $\left(-W_{k}\right)$, and $\mathbf{I}=\Gamma_{\bullet k} \Phi_{k}$ is the matrix of that conductor currents for which the fluxes per unit length trough the $\left(-W_{l}\right)$ for $l \in\{1, \ldots, n\} \backslash\{k\}$ are vanishing and that one trough $\left(-W_{k}\right)$ is equal to $\Phi_{k}$ where $\boldsymbol{\Gamma}_{\bullet k}$ denotes the $k$ th column of $\boldsymbol{\Gamma}$.

It can be shown (Jost, 2002) that for sufficiently smooth conductor surfaces all the functions exist and that they fulfill the assumptions necessary to applicate results of of the theory of planar fields.

The symmetry of the matrices $\mathbf{C}$ and $\mathbf{L}$ can be proved by means of a variant of the Green's integral theorems specialized for planar fields. The positive definiteness of these matrices follows from the positive definiteness of the energy stored in the electric and magnetic field of $\Lambda$.

\section{Concluding remarks}

Based on Wagner (1914); Abraham and Föppl (1917); Adler et al. (1960); Helm (1975), we have presented in Reibiger (2002) a thorough field theoretic approach for the description of the propagation of TEM waves on lossless multiconductor transmission lines by means of the system of transmission line equations. Together with the remarks guaranteeing the 
existence of the matrices of capacitance and inductance coefficients for such transmission lines from that approach it follows also the existence of TEM waves on lossless multiconductor lines.

I would like to thank Prof. Dr. G. Helm (formerly TU Chemnitz), Prof. Dr. J. Nitsch (Otto von Guericke Universität Magdeburg), and esspecially Dipl.-Ing. T. Nähring (TU Dresden) for their interest, inspiring discussions, and critical remarks.

\section{References}

Abraham, M. and Föppl, A.: Theorie der Elektrizität, Volume I, B. G. Teubner, Leipzig, 5th edition, 1917.

Adler, R. B., Chu, L. J., and Fano, R. M.: Electromagnetic energy, transmission and radiation, J. Wiley, New York, 1960.

Heaviside, O.: Electrical papers, Volume I, Chelsea Publ.C., 2nd Edition, 1970.
Heaviside, O.: Electromagnetic theory, Chelsea Publ.C., New York, 3rd Edition, 1971.

Helm, G.: Das dynamische Verhalten realer Mehrfachleitungen, Habilit., TH Karl-Marx-Stadt, 1975.

Henrici, P.: Applied and computational analysis, J. Wiley, New York, 1974.

Jost, J.: Partial differential equations, Springer, New York, 1st Edition, 2002.

Lawrentjew, M. A. and Schabat, B. W.: Methoden der komplexen Funktionentheorie, Deutscher Verlag der Wissenschaften, Berlin, 1967.

Nähring, T.: Nichttransversale Felder in Kabelmodellen, In TU preprint, TU Dresden, in preparation, 2002.

Reibiger, A.: Field theoretic description of tem waves in multiconductor transmission lines, In I. Canavero, F. Maio, editor, Proc. 6th IEEE Workshop on Signal Propagation on Interconnects, pages 93-96, Torino, Politecnico di Torino, 2002.

Wagner, K. W.: Induktionswirkungen von Wanderwellen in Nachbarleitungen, ETZ, 35:639-643, 677-680, 705-708, 1914. 